\title{
Púrpura Trombocitopênica e Anemia Hemolítica Auto-Imune em Pacientes Internados com Lúpus Eritematoso Sistêmico Juvenil
}

\section{Trombocytopenic Purpura and Autoimmune Hemolytic Anemia in Hospitalized Patients with Juvenile Systemic Lupus Erythematosus}

\author{
Jochebed Kyoung Kim ${ }^{(1)}$, Mércia Maria Moreira Facó(2), Ana Paola Navarette Lotito ${ }^{(3)}$, Bernadete Lourdes \\ Liphaus $^{(3)}$, Jorge David Aivazoglou Carneiro ${ }^{(4)}$, Clovis Artur Almeida Silva $^{(5)}$
}

\begin{abstract}
RESUMO
Objetivo: avaliar o comprometimento hematológico $(\mathrm{CHE})$ em pacientes internados com lúpus eritematoso sistêmico juvenil (LESJ). Métodos: durante o período de 1994 a 2005, ocorreram 195 internações de 77 pacientes com LESJ (critérios do Colégio Americano de Reumatologia) e foram acompanhadas pela Unidade de Reumatologia Pediátrica do Instituto da Criança - Universidade de São Paulo. Essas internações foram avaliadas com relação à presença de CHE inicial ou evolutivo: anemia hemolítica auto-imune ou púrpura trombocitopênica. Todos os pacientes realizaram dois ou mais hemogramas. O diagnóstico de anemia hemolítica auto-imune (AHAI) foi estabelecido pela queda acentuada dos níveis de hemoglobina (acima de $2 \mathrm{~g} / \mathrm{dl}$ ), reticulocitose, aumento de desidrogenase láctica (DHL), aumento de bilirrubina indireta e teste de Coombs positivo. As manifestações hematológicas associadas à infecção, neoplasia e anemia aplásica foram excluídas. Resultados: o CHE ocorreu em 14 pacientes (18,9\%), com 15 internações. Destes, 11 eram do sexo feminino, sete apresentaram púrpura trombocitopênica, cinco, anemia hemolítica auto-imune e dois, síndrome de Evans. O CHE, como manifestação inicial e isolada do LESJ, foi evidenciado em três pacientes. Todos os pacientes com púrpura trombocitopênica apresentaram sangramento cutâneo (petéquias e/ou equimoses). Todos estavam em atividade da doença e apresentavam simultaneamente outras manifestações do LESJ, particularmente nefrite e vasculite. Inicialmente, todos receberam pulsoterapia com metilprednisolona e, posteriormente, prednisona. Em três pacientes, o tratamento foi predominantemente realizado para controle das manifestações hematológicas, com gamaglobulina endovenosa. Os imunossupressores mais utilizados foram pulsoterapia endovenosa com ciclosfosfamida, ciclosporina e azatioprina. Óbito ocorreu em uma paciente por sangramento de sistema nervoso central. Nenhum paciente necessitou de esplenectomia. Conclusões: o CHE isolado foi uma manifestação grave em pacientes internados com LESJ, habitualmente associado a uma doença ativa e sistêmica.
\end{abstract}

Palavras-chave: lúpus eritematoso sistêmico, criança, adolescente, trombocitopenia, anemia hemolítica auto-imune.

\begin{abstract}
Objective: to evaluate the hematological involvement (HI) in hospitalized patients with juvenile systemic lupus erythematosus (JSLE). Methods: from 1994 to 2005, 195 admissions occurred in 77 JSLE patients (American College of Rheumatology criteria) and were followed by the Pediatric Rheumatology Unit of the Instituto da Criança-University of São Paulo. These admissions were evaluated according to the presence of HI at onset or during the evolution of the disease: autoimmune hemolytic anemia (AHA) or thrombocytopenic purpura. All patients performed at least two complete blood counts. AHA was defined by a fall in hemoglobin levels (beyond $2 \mathrm{~g} / \mathrm{dl}$ ), reticulocytosis, increase in lactate dehydrogenase $(\mathrm{LDH})$ and indirect bilirubin levels, and a positive Coombs test. The hematologic manifestations associated with infection, neoplasia and aplastic anemia were excluded. Results: HI occurred in 14 patients (18.9\%), with 15 admissions. Among these patients, 11 were female, 7 had trombocytopenic purpura, $5 \mathrm{AHA}$ and 2 Evans syndrome. $\mathrm{HI}$ as onset and single manifestation of JSLE was observed in three patients. All the patients with trombocytopenic purpura presented cutaneous bleeding (petechia and/or ecchymosis). All had disease activity and simultaneously presented other manifestations of JSLE, particularly nephritis and vasculitis. Initially, all patients received pulsetherapy with methylprednisolone and prednisone later. In three patients the treatment aimed predominantly the control of hematologic manifestations, with intravenous gammaglobulin. The most used immunossupressive therapies were intravenous cyclophosphamide, cyclosporine and azathioprine. One patient died of central nervous system bleeding. No patient needed splenectomy. Conclusions: isolated HI was a severe manifestation in hospitalized patients with JSLE, generally associated with an active and systemic disease.
\end{abstract}

Keywords: systemic lupus erythematosus, child, adolescent, trombocytopenia and autoimmune hemolytic anemia.

\footnotetext{
Unidades de Reumatologia e Hematologia Pediátricas - Instituto da Criança do Hospital das Clínicas da Faculdade de Medicina da Universidade de São Paulo (ICr-HC-FMUSP). Recebido em 2/9/2006. Aprovado, após revisão, em 02/1 1/2006.

1. Aluna de Graduação da FMUSP e de Iniciação Científica do Departamento de Pediatria da FMUSP.

2. Mestre em Ciências pela FMUSP.

3. Doutor em Ciências pela FMUSP. Médica assistente da Unidade de Reumatologia Pediátrica do Departamento de Pediatria da FMUSP.

4. Doutor em Ciências pela FMUSP. Chefe da Unidade de Hematologia Pediátrica do Departamento de Pediatria da FMUSP.

5. Doutor em Medicina pela FMUSP. Chefe da Unidade de Reumatologia Pediátrica do Departamento de Pediatria da FMUSP.

Endereço para correspondência: Clovis Artur Almeida da Silva, Rua Senador Cesar Lacerda Vergueiro, 494/82, CEP 05435-010, São Paulo, SP, Brasil, telefone: (11) 3069-8675, fax: (11) 3069-8510, e-mail: clovisaas@icr.hcnet.usp.br
} 


\section{INTRODUÇÃO}

O lúpus eritematoso sistêmico (LES) juvenil (LESJ) é uma doença rara, cujo início pode ser abrupto ou insidioso, com manifestações clínicas variáveis, dependendo do órgão ou sistema envolvido ${ }^{(1)}$. Crianças e adolescentes com LESJ habitualmente apresentam início da doença e curso evolutivo mais graves em relação a pacientes adultos com lúpus eritematoso sistêmico (LES) ${ }^{(2)}$.

O comprometimento hematológico no LESJ, particularmente anemia hemolítica auto-imune (AHAI) e púrpura trombocitopênica, pode estar associado à atividade da doença com produção de auto-anticorpos (anticorpos anti-hemácias, antiplaquetas e antifosfolípides $)^{(2,3,4)}$, ao uso de medicamentos utilizados para o controle desta, como pulsoterapia com ciclofosfamida endovenosa ${ }^{(5)}$, e às infecções ${ }^{(6,7)}$.

As alterações hematológicas (trombocitopenia, AHAI, leucopenia e/ou linfopenia) constituem um dos critérios de classificação para o diagnóstico de LESJ segundo o American College of Rheumatology (ACR) ${ }^{(8)}$, e esses comprometimentos hematológicos podem ser a primeira manifestação da doença e anteceder o diagnóstico de LESJ em meses a anos. Adicionalmente, essas manifestações (isoladas ou associadas a outros envolvimentos da doença) são consideradas fator de gravidade e de pior prognóstico ${ }^{(9-12)}$, sendo associadas com aumento de mortalidade, particularmente por sangramento do sistema nervoso central (SNC) ${ }^{(11)}$.

A escassez de estudos, avaliando predominantemente esses envolvimentos nos pacientes internados com LESJ, e a expressiva casuística dessa doença no presente Serviço de Reumatologia Pediátrica estimularam a realização dessa pesquisa.

O objetivo do presente trabalho foi avaliar o comprometimento hematológico (púrpura trombocitopênica e/ou AHAI) em pacientes internados com LESJ.

\section{PACIENTES E MÉTODO}

Durante o período de 12 anos (janeiro de 1994 a dezembro de 2005), ocorreram 195 internações de 77 pacientes com LESJ e foram acompanhadas pelas Unidades de Reumatologia e Hematologia Pediátricas do Instituto da Criança (ICr) do Hospital das Clínicas (HC) da Faculdade de Medicina da Universidade de São Paulo (FMUSP).

Todos os pacientes preencheram os critérios de classificação para o diagnóstico de LES segundo o $\mathrm{ACR}^{(8)} \mathrm{e}$ tiveram idade de início da doença inferior a 18 anos, caracterizando o início juvenil. No momento da internação, todos os pacientes estudados apresentaram comprometimento hematológico e foram admitidos nos seguintes locais do nosso Hospital Universitário: enfermaria de especialidade, pronto-socorro, unidade semi-intensiva ou unidade de terapia intensiva.

O presente estudo foi uma série de casos. As internações foram estudadas com relação ao comprometimento hematológico (púrpura trombocitopênica e/ou AHAI) e incluíram avaliação dos dados demográficos, características clínicas, atividade e dano cumulativo da doença, exames laboratoriais e terapias utilizadas para o LESJ.

A atividade do LESJ foi avaliada pelo Systemic Lupus Erithematosus Disease Activity Index (Sledai) ${ }^{(13)}$, sendo considerados arbitrariamente como atividade da doença valores numéricos acima de um. $\mathrm{O}$ dano cumulativo irreversível causado pelo LESJ e /ou tratamento utilizado foi determinado pelo Systemic Lupus International Collaborating Clinics/ACR (Slicc-ACR) Damage Index (Slicc-ACR/ $\mathrm{DI})^{(14)}$. Valores desse escore igual ou acima de um, avaliados após seis meses de duração da doença, foram considerados como presença de dano cumulativo da doença. Os escores Sledai e Slicc-ACR/DI foram avaliados em todos os pacientes no início da internação, retrospectivamente.

Com relação ao comprometimento hematológico, AHAI foi apenas definida pela presença dos cinco critérios seguintes: queda acentuada dos níveis de hemoglobina (acima de $2 \mathrm{~g} / \mathrm{dl}$ ), reticulocitose, aumento de DHL, aumento de bilirrubina indireta e teste de Coombs positivo; púrpura trombocitopênica foi definida pela presença de sangramento e trombocitopenia (plaquetas abaixo de $100.000 / \mathrm{mm}^{3}$ por um período mínimo acima de um mês); leucopenia e linfopenia com leucócitos e linfócitos abaixo de $4.000 \mathrm{~mm}^{3}$ e $1.500 \mathrm{~mm}^{3}$, respectivamente. Síndrome de Evans correspondeu à associação de púrpura trombocitopênica e AHAI. Para confirmação dessas alterações hematológicas, foram realizados em todos os pacientes pelo menos dois hemogramas completos. Manifestações hematológicas associadas a infecções (como sepse), neoplasia e/ou anemia aplásica foram excluídas.

Os seguintes anticorpos antifosfolípides foram avaliados em todos os pacientes durante a internação: anticorpos anticardiolipina isotipos $\operatorname{IgM}$ e $\operatorname{IgG}$ (avaliados pela técnica de Elisa) e anticoagulante lúpico (avaliado por tempo de tromboplastina parcial ativado diluído, tempo de coagulação de Kaolin e tempo do veneno de víbora de Russel $)^{(3)}$. Retrospectivamente, o diagnóstico de síndrome do anticorpo antifosfolípide (SAF) foi estabelecido de acordo com o consenso internacional de classificação 
da síndrome do anticorpo antifosfolípide. Essa síndrome foi definida pela presença de trombose vascular (arterial, venosa ou trombose de vasos de pequeno calibre, em qualquer tecido ou órgão) ou pela presença de morbidade gestacional [um ou mais abortamentos (antes de dez semanas, com morfologia fetal normal à ultra-sonografia), prematuridade (antes de 34 semanas, com recém-nascido normal por eclâmpsia ou insuficiência placentária) ou três ou mais abortamentos espontâneos, inexplicáveis e consecutivos (antes de dez semanas com alterações hormonais ou anatômicas maternas)], associada à positividade de um ou mais dos anticorpos antifosfolípides, em pelo menos duas ocasiões com intervalo mínimo de 12 semanas. Os anticorpos anticardiolipina isotipos $\operatorname{IgM}$ e $\operatorname{IgG}$ devem apresentar títulos de moderados a altos (acima de 40 GPL ou MPL) ${ }^{(4)}$.
Esse projeto de iniciação científica do Departamento de Pediatria da FMUSP foi aprovado pelo Comitê de Pesquisa e Ética do HC-FMUSP (CAPPesq número 520/05).

\section{RESULTADOS}

Das 195 internações que ocorreram nesses 12 anos, 15 motivaram as hospitalizações por comprometimento hematológico $(7,7 \%)$ e ocorreram em 14 dos 77 pacientes $(18,9 \%)$ Desses 14 pacientes, 11 eram do sexo feminino, sete apresentaram púrpura trombocitopênica, cinco tiveram AHAI e dois, associação dessas duas entidades (síndrome de Evans). Leucopenia e linfopenia ocorreram em cinco pacientes. A Tabela 1 inclui dados demográficos, manifestações clínicas, exames laboratoriais e tratamento dos pacientes internados com LESJ e comprometimento hematológico.

TABELA 1

DADOS DEMOGRÁFICOS, MANIFESTAÇÕES CLÍNICAS, EXAMES LABORATORIAIS E TRATAMENTO DOS PACIENTES INTERNADOS COM LÚPUS ERITEMATOSO SISTÊMICO JUVENIL (LESJ) E COMPROMETIMENTO HEMATOLÓGICO

\begin{tabular}{|c|c|c|c|c|c|c|c|c|}
\hline Caso & Sexo & Id (a) & IC (a) & $\Delta \mathrm{T}(\mathrm{a})$ & $\begin{array}{l}\text { Sledai/ } \\
\text { Slicc- } \\
\text { ACR/DI }\end{array}$ & $\mathbf{P} / \mathbf{A}$ & Tratamento & $\begin{array}{l}\text { Manifestações e exames } \\
\text { laboratoriais do LESJ }\end{array}$ \\
\hline 1 & $\mathrm{~F}$ & 7 & 8,3 & 1,3 & $12 / 0$ & $\mathrm{~S} / \mathrm{N}$ & $\mathrm{Pd}, \mathrm{PMP}, \mathrm{PC}$ & $\begin{array}{l}\text { Nefrite } \mathrm{V} \text {, convulsão, artrite, } \\
\text { vasculite, anti-DNA }\end{array}$ \\
\hline 2 & $\mathrm{~F}$ & 7,8 & 8 & 0,2 & $8 / N R$ & $\mathrm{~N} / \mathrm{S}$ & $\mathrm{Pd}, \mathrm{PMP}, \mathrm{Cl}$ & $\begin{array}{c}\text { Convulsão, artrite, vasculite, } \\
\text { leuco/linfopenia, anti-DNA }\end{array}$ \\
\hline 3 & $\mathrm{~F}$ & 12,5 & 12,5 & 0 & $14 / N R$ & $\mathrm{~S} / \mathrm{N}$ & $\mathrm{Pd}, \mathrm{PMP}, \mathrm{Aza}, \mathrm{Cl}$ & Nefrite, vasculite \\
\hline 4 & $\mathrm{~F}$ & 13,5 & 13,5 & 0 & $2 / N R$ & $\mathrm{~N} / \mathrm{S}$ & $\mathrm{Pd}, \mathrm{PMP}, \mathrm{Cl}$ & Nefrite classe IV, vasculite \\
\hline 5 & $\mathrm{~F}$ & 9,2 & 9,5 & 0,3 & $14 / N R$ & $\mathrm{~N} / \mathrm{S}$ & $\mathrm{Pd}, \mathrm{PMP}, \mathrm{PC}, \mathrm{Cl}$ & Nefrite classe IV, artrite, vasculite \\
\hline 6 & $\mathrm{~F}$ & 12,3 & 10,3 & -2 & $4 / N R$ & $\mathrm{~N} / \mathrm{S}$ & $\begin{array}{l}\text { Pd, PMP, GGEV, } \\
\text { PC, AC }\end{array}$ & $\begin{array}{l}\text { Nefrite classe III, pleurite, AAF } \\
\text { (Acl IgG } 80 \mathrm{GPL} \text { e AL), SAF, } \\
\text { leuco/linfopenia, anti-Sm }\end{array}$ \\
\hline 7 & M & 4,4 & 4,5 & 0,1 & $2 / N R$ & $\mathrm{~S} / \mathrm{N}$ & $\mathrm{Pd}, \mathrm{PMP}, \mathrm{PC}, \mathrm{Cl}$ & $\begin{array}{l}\text { Nefrite classe III, vasculite, } \\
\text { artrite, pericardite }\end{array}$ \\
\hline 8 & $\mathrm{~F}$ & 13,7 & 14,1 & 0,4 & $26 / N R$ & $\mathrm{~S} / \mathrm{N}$ & $\begin{array}{l}\mathrm{Pd}, \mathrm{PMP}, \mathrm{PC}, \\
\text { plasmaferese }\end{array}$ & $\begin{array}{l}\text { Nefrite classe IV, psicose, vasculite, } \\
\text { AAF (Acl IgM } 46 \text { MPL), anti-DNA }\end{array}$ \\
\hline 9 & $\mathrm{~F}$ & 11,5 & 16,8 & 5,3 & $8 / 0$ & $\mathrm{~N} / \mathrm{S}$ & $\mathrm{Pd}, \mathrm{PMP}, \mathrm{PC}, \mathrm{Cl}$ & $\begin{array}{c}\text { Nefrite classe III, artrite, vasculite, } \\
\text { leuco/linfopenia }\end{array}$ \\
\hline 10 & M & 14,9 & 11,9 & -3 & $12 / N R$ & $S / S$ & Pd, PMP, PC, Ac & $\begin{array}{c}\text { Nefrite classe V, AAF } \\
(\text { Acl IgG } 60 \mathrm{GPL} \text { e } \mathrm{AL}), \mathrm{SAF} \text {, anti-Sm }\end{array}$ \\
\hline 11 & M & 11 & 16,1 & 5,1 & $3 / 2$ & $\mathrm{~S} / \mathrm{N}$ & $\begin{array}{l}\text { Pd, PMP, PC, } \\
\text { GGEV, Dapsona, } \\
\text { ciclosporina }\end{array}$ & $\begin{array}{l}\text { Convulsão, vasculite intestinal, } \\
\text { pericardite leuco/linfopenia, anti-DNA }\end{array}$ \\
\hline 12 & $\mathrm{~F}$ & 7,7 & 11 & 3,3 & $12 / 0$ & $\mathrm{~S} / \mathrm{N}$ & $\mathrm{Pd}, \mathrm{PMP}, \mathrm{PC}$ & Nefrite classe IV, vasculite, artrite, anti-DNA \\
\hline 13 & $\mathrm{~F}$ & 9,8 & 19,5 & 9,7 & $12 / 1$ & $S / S$ & $\mathrm{Pd}, \mathrm{PMP}, \mathrm{Aza}$ & Nefrite classe V, vasculite, artrite \\
\hline 14 & $\mathrm{~F}$ & 12,1 & 11,1 & -1 & $8 / 0$ & $\mathrm{~S} / \mathrm{N}$ & $\begin{array}{l}\text { Pd, PMP, Cl GGEV, } \\
\text { ciclosporina }\end{array}$ & $\begin{array}{c}\text { Convulsão, vasculite, artrite, úlcera de } \\
\text { mucosas leuco/linfopenia anti-DNA }\end{array}$ \\
\hline
\end{tabular}

F - sexo feminino, M - sexo masculino, Id - idade ao diagnóstico da doença, IC - idade do comprometimento hematológico, $\Delta \mathrm{T}$ - intervalo de tempo entre o comprometimento hematológico e o diagnóstico do LESJ, a - anos, Sledai -Systemic Lupus Erithematosus Disease Activity índex, Slicc-ACR/DI - Systemic Lupus International Collaborating Clinics/ACR Damage index, P - púrpura trombocitopênica, A - anemia hemolítica, S - sim, N - não, AAF - anticorpo antifosfolípide, $\mathrm{Acl}$ - anticorpo anticardiolipina, AL - anticoagulante lúpico, SAF - síndrome do anticorpo antifosfolípide, NR - não-realizado, Pd - prednisona, Cl -cloroquina, PC - pulsoterapia com ciclofosfamida, PMP - pulsoterapia com metilprednisolona, Aza - azatioprina, MTX - metotrexato, Ac -anticoagulante (heparina e dicumarínico), GGEV - gamaglobulina endovenosa. 
A média de idade ao diagnóstico dos 14 pacientes com LESJ foi de 10,5 anos (variação de 4,4 a 14,9 anos) e a média da idade do comprometimento hematológico foi de 11,9 anos (variação de 4,5 a 19,5 anos).

O comprometimento hematológico, como manifestação inicial e isolada do LESJ, foi evidenciado em três pacientes: AHAI em um (caso 6), púrpura trombocitopênica imunológica (PTI) crônica, com duração de um ano, em um (caso 14) e síndrome de Evans em outro (caso 10). Os três pacientes realizaram mielograma que evidenciou hiperplasia das séries megacariocítica e/ou eritroblástica. Nesses três pacientes, a mediana do intervalo de tempo entre o início do comprometimento hematológico e o diagnóstico do LESJ foi de 24 meses (variação de 12 a 36 meses). Em dois pacientes, o comprometimento hematológico ocorreu no momento do diagnóstico e esteve associado a outras manifestações da doença (casos 3 e 4). Por sua vez, em nove pacientes, esse comprometimento foi evidenciado após o diagnóstico da doença, com mediana de 39 meses (variação de dois a 117 meses).

Com relação à presença de sangramentos, petéquias e/ou equimoses, estas ocorreram em todos os pacientes, gengivorragia em cinco pacientes, sangramento gastrintestinal (hematêmese ou melena) em três e epistaxe ocorreu em três.

Associação do comprometimento hematológico a outras manifestações do LESJ foi evidenciada em todos os pacientes: nefrite em 11 , vasculite em 12 (cutânea em 11 e intestinal em um), artrite em sete, envolvimento de SNC em cinco e serosite (pleurite e/ou pericardite) em três.

No momento da internação, foi observada atividade da doença em todos os pacientes e a mediana do Sledai desses 14 pacientes foi de dez (variação de dois a 26). Dano cumulativo do LESJ ocorreu em dois pacientes, um apresentou catarata e infarto intestinal com necessidade de ressecção duodenal e o outro, catarata (casos 11 e 13).

Os anticorpos antifosfolípides foram evidenciados em três dos 14 pacientes, sendo os anticorpos anticardiolipina isotipo IgG presentes em todos (casos 6, 8 e 10) e anticoagulante lúpico em dois (casos 6 e 10). O diagnóstico de SAF foi estabelecido em dois pacientes: um na sua primeira internação com trombose venosa profunda dos membros inferiores logo após o diagnóstico (caso 10) e outro ocorreu cinco anos após o diagnóstico do LESJ em uma paciente que iniciou a doença com AHAI e apresentou acidente vascular cerebral isquêmico (caso 6).

O tratamento do LESJ foi priorizado de acordo com as manifestações da doença. Inicialmente, todos os pacientes receberam pulsoterapia com metilprednisolona e, posterior- mente, prednisona. Os imunossupressores mais utilizados foram pulsoterapia endovenosa com ciclosfosfamida, ciclosporina e azatioprina. Em três pacientes, o tratamento foi predominantemente realizado para controle das manifestações hematológicas (casos 6, 11 e 14). Estes receberam gamaglobulina endovenosa $(2 \mathrm{~g} / \mathrm{kg} /$ mês divididos em duas doses, com intervalo de 24 horas). A púrpura trombocitopênica do caso 11 apenas remitiu com associação de gamaglobulina endovenosa mensal, danazol e ciclosporina. Apesar da terapêutica administrada no caso 14, o paciente evoluiu para óbito após sangramento de SNC. Nenhum dos pacientes necessitou de esplenectomia.

\section{DISCUSSÃO}

Comprometimento hematológico inicial ou evolutivo tem sido descrito em 39\% das crianças e adolescentes com LESJ: trombocitopenia em $8 \%$ a $74 \%$, anemia em $72 \%$ a $84 \%$, anemia hemolítica em $6 \%$ a $10 \%$, leucopenia em $27 \%$ a $52 \%$ e linfopenia em $30 \%$ a $59 \%^{(1,2,9-12)}$.

O LESJ é mais grave em relação ao LES dos adultos e alguns autores evidenciaram um maior predomínio de manifestações hematológicas na população pediátrica ${ }^{(2,10,15)}$. Rood et $\mathrm{al}^{\left({ }^{(10)}\right.}$ avaliaram 31 pacientes com LESJ e $135 \mathrm{com}$ LES. Os pacientes com LESJ apresentaram maior freqüência de leucopenia evolutiva (74\% versus $50 \%)$ e trombocitopenia ( $48 \%$ versus $36 \%$ ). Por sua vez, Costallat et al ${ }^{(15)}$ estudaram 75 pacientes com LESJ versus 393 com LES e evidenciaram que a trombocitopenia esteve relacionada ao óbito em 66\% dos LESJ e 58\% dos LES.

A presente casuística incluiu apenas pacientes internados que apresentavam doença ativa, habitualmente com envolvimento de vários órgãos e sistemas, com necessidade de uso de medicação endovenosa, particularmente pulsoterapia com metilprednisolona. Uma limitação do delineamento dessa pesquisa foi o possível viés da amostra estudada, com inclusão de pacientes internados e, por isso, com maior chance de se encontrarem em atividade da doença e com manifestações potencialmente graves.

Poucos estudos enfocaram preferencialmente as manifestações hematológicas, como AHAI e púrpura trombocitopênica ${ }^{(11,12)}$. Schmugge et a $l^{(11)}$ estudaram 106 pacientes ambulatoriais e internados com LESJ. Destes, 34\% apresentaram trombocitopenia com valores inferiores a 100.000 $\mathrm{mm}^{3}$. Onze por cento apresentaram o diagnóstico inicial de PTI e 1\%, de síndrome de Evans. Braga et al ${ }^{(12)}$ descreveram cinco casos do sexo feminino com diagnóstico de PTI que desenvolveram LESJ, em média um ano e dez meses após 
o início da trombocitopenia. Na presente casuística houve um predomínio de adolescentes do sexo feminino (57\%), cujas manifestações hematológicas ocorreram, em geral, na evolução da doença. Apenas três casos (4\%) apresentaram manifestações hematológicas como manifestação inicial do LESJ, sendo um com PTI, um com AHAI e outro com síndrome de Evans.

A PTI e a AHAI são doenças auto-imunes caracterizadas por destruição extravascular aumentada de plaquetas e hemácias, causadas por auto-anticorpos antiplaquetários ou antihemácias, os quais se ligam às plaquetas e hemácias circulantes, resultando em destruição pelo sistema retículo-endotelial ${ }^{(12,16)}$. Alguns autores recomendam a realização de auto-anticorpos em todos os pacientes com PTI para excluir o diagnóstico de LESJ, particularmente naqueles do sexo feminino e com a forma crônica da doença ${ }^{(17,18)}$. Entretanto, existem pacientes com PTI e positividade para o fator antinúcleo que não irão preencher, ou apenas posteriormente preencherão, os critérios de classificação para o diagnóstico de $\operatorname{LES}^{(19)}$.

Um outro aspecto interessante é que os anticorpos antifosfolípides podem ser parcialmente responsáveis pela trombocitopenia relacionada ao LESJ, em decorrência dos anticorpos contra fosfolípides na membrana plaquetária $^{(3,4,12)}$, conforme ocorreu em dois de nossos pacientes. No atual critério de classificação da SAF, os autores ressaltam que na presença de trombocitopenia em pacientes com anticorpos antifosfolípides persistentemente positivos, mesmo na ausência de manifestações clínicas de SAF, estes não devem ser considerados como PTI e devem ser monitorizados rigorosamente pelo maior risco evolutivo de tromboses ${ }^{(4)}$.

O prognóstico e a mortalidade da púrpura trombocitopênica estão relacionados aos sangramentos, particularmente hemorragia do SNC, como ocorreu em um caso deste estudo. Schmugge et $a l^{(11)}$ evidenciaram sangramentos em

\section{REFERÊNCIAS}

1. Hilário MO, Len CA, Sato EI, et al: Lúpus eritematoso sistêmico juvenil: resultados do estudo multicêntrico nacional. Rev Bras Reumatol 42: 85-9, 2002.

2. Stichweh D, Arce E, Pascual V: Update on pediatric systemic lupus erythematosus. Curr Opin Rheumatol 16: 577-87, 2004.

3. Campos LM, Kiss MH, D'Amico EA, Silva CA: Antiphospholipid antibodies and antiphospholipid syndrome in 57 children and adolescents with systemic lupus erythematosus. Lupus 12: 8206, 2003.

4. Miyakis S, Lockshin MD, Atsumi T, et al: International consensus statement on an update of the classification criteria for definite antiphospholipid syndrome (APS). J Thromb Haemost 4: 295 306,2006
11\% dos pacientes com LES e púrpura trombocitopênica, sendo que 3\% foram por hemorragia de SNC.

No presente estudo, o envolvimento hematológico ocorreu simultaneamente com atividade da doença e outras manifestações desta, particularmente nefrite e vasculite, de acordo com outros estudos da literatura ${ }^{(1,2,10)}$.

A opção do uso de imunossupressores para o LESJ foi individualizada para cada paciente de acordo com: literatura médica, experiência anterior da Unidade de Reumatologia Pediátrica do ICr-HC-FMUSP e disponibilidade do medicamento no momento de sua introdução. A maioria dos estudos são casuísticas isoladas e indicam o uso de corticosteróide e gamaglobulina endovenosa para os pacientes que apresentem predominantemente púrpura trombocitopência ou $\mathrm{AHAI}^{(11)}$. Nos casos refratários, imunossupressores são indicados conforme os outros comprometimentos do LESJ (como nefrite e envolvimento neuropsiquiátrico), particularmente pulsoterapia com ciclofosfamida, azatioprina ou ciclosporina ${ }^{(20)}$.

Recentemente, a terapia com anticorpo monoclonal quimérico anti-CD20 (rituximabe), com redução significativa dos linfócitos B, tem sido segura e eficaz no LES $^{(21)}$ e LESJ $^{(22,23)}$, podendo ser alternativa para os casos resistentes com as terapias anteriores.

Assim sendo, o comprometimento hematológico foi uma manifestação grave em pacientes internados com LESJ, habitualmente associado à doença ativa, com comprometimento sistêmico e evolutivo.

\section{AGRADECIMENTO}

Este estudo foi patrocinado pelo Conselho Nacional de Desenvolvimento Científico e Tecnológico - CNPq (número 302469/2005-2 para CAAS).

\section{Declaramos a inexistência de conflitos de interesse.}

5. Lehman TJ, Onel K: Intermittent intravenous cyclophosphamide arrests progression of the renal chronicity index in childhood systemic lupus erythematosus. J Pediatr 136: 243-7, 2000.

6. Len CA, Terreri MT, Hilário MO: Lúpus eritematoso sistêmico juvenil e infecção. Rev Bras Reumatol 42: 218-22, 2002.

7. Canova EG, Rosa DC, Vallada MG, Silva CA: Invasive aspergillosis in juvenile systemic lupus erythematosus. A clinicopathologic case. Clin Exp Rhematol 20: 736, 2002.

8. Hochberg MC: Updating the American College of Rheumatology revised criteria for the classification of systemic lupus erythematosus. Arthritis and Rheumatism 40: 1725, 1997.

9. Iqbal S, Sher MR, Good RA, Cawkwell GD: Diversity in presenting manifestations of systemic lupus erythematosus in children. J Pediatr 135: 500-5, 1999. 
10. Rood MJ, ten Cate R, van Suijlekom-Smit LW, et al: Childhoodonset Systemic Lupus Erythematosus: clinical presentation and prognosis in 31 patients. Scand J Rheumatol 28: 222-6, 1999.

11. Schmugge M, Revel-Vilk S, Hiraki L, Rand ML, Blanchette VS, Silverman ED: Thrombocytopenia and thromboembolism in pediatric systemic lupus erythematosus. J Pediatr 143: 666-9, 2003.

12. Braga JA, Hokazono M, Tererri MT, Hilário MO: Púrpura trombocitopênica imunológica com manifestação inicial de lúpus eritematoso sistêmico juvenil. Rev Bras Reumatol 43: 392-6, 2003.

13. Brunner HI, Silverman ED, To T, Bombardier C, Feldman BM: Sensitivity of the Systemic Lupus Erythematosus Disease Activity Index, British Isles Lupus Assessement Group Index, and Systemic Lupus Activity Measure in the evaluation of clinical change in childhood-onset systemic lupus erythematosus. Arthritis and Rheumatism 42: 1354-60, 1999.

14. Brunner HI, Silverman ED, To T, Bombardier C, Feldman BM: Risk factors for damage in childhood-onset systemic lupus erythematosus. Arthritis and Rheumatism 46: 436-4, 2002.

15. Costallat LT, Appenzeller S, Marini R: Evolução e fatores prognósticos do lúpus eritematoso sistêmico em relação com a idade de início. Rev Bras Reumatol 42: 91-8, 2002.

16. Waters AH: Autoimmune thrombocytopenia: clinical aspects. Semin Hematol 29: 18-25, 1992.
17. Anderson MJ, Peebles CL, McMillan R, Curd JG: Fluorescent antinuclear antibodies and anti-SS-A/Ro in patients with immune thrombocytopenia subsequently developing systemic lupus erythematosus. Ann Intern Med 103: 548-50, 1985.

18. Zimmerman SA, Ware RE: Clinical significance of the antinuclear antibody test in selected children with idiopathic thrombocytopenic purpura. J Pediatr Hematol Oncol 19: $297-$ 303, 1997.

19. Panzer S, Penner E, Graninger W, Schulz E, Smolen JS: Antinuclear antibodies in patients with chronic idiopathic autoimmune thrombocytopenia followed 2-30 years. Am J Hematol 32: 100-3, 1989.

20. Silva CA: Doenças refratárias ao tratamento convencional: como proceder? (Parte 2). Lúpus eritematoso sistêmico Juvenil: nefrite e envolvimento neuropsiquiátrico. Rev Bras Reumatol 44 (Supl. 1): 41-3, 2004.

21. Looney RJ, Anolik JH, Campbell D, et al: B cell depletion as a novel treatment for systemic lupus erythematosus: a phase I/II doseescalation trial of rituximab. Arthritis Rheum 50: 2580-9, 2004.

22. ten Cate R, Smiers FJ, Bredius RG, et al: Anti-CD20 monoclonal antibody (rituximab) for refractory autoimmune thrombocytopenia in a girl with systemic lupus erythematosus. Rheumatology (Oxford) 43: 244, 2004.

23. Binstadt BA, Caldas AM, Turvey SE, et al: Rituximab therapy for multisystem autoimmune diseases in pediatric patients. J Pediatr 14: 598-604, 2003 